Rapid Reviews COVID-19

\title{
Review 2: "Robust SARS- \\ CoV-2-specific T-cell \\ immunity is maintained at \\ 6 months following \\ primary infection"
}

Sulggi Lee ${ }^{1}$

${ }^{1}$ University of California San Francisco School of Medicine, Medicine

Published on: Jan 17, 2021

DOI: $10.1162 / 2$ e3983f5.cc0c1de1

License: Creative Commons Attribution 4.0 International License (CC-BY 4.0). 


\section{$\underline{\text { RR:C19 Evidence Scale rating by reviewer: }}$}

- Reliable. The main study claims are generally justified by its methods and data. The results and conclusions are likely to be similar to the hypothetical ideal study. There are some minor caveats or limitations, but they would/do not change the major claims of the study. The study provides sufficient strength of evidence on its own that its main claims should be considered actionable, with some room for future revision.

\section{Review:}

The authors studied 100 COVID+ mild-moderate (44\% asymptomatic) patients and found that virusspecific (to spike and non-spike proteins) CD4 and CD8 T cell responses were still present at 6 months after infection. $\mathrm{T}$ cell responses were higher with symptomatic disease, with $\mathrm{CD} 4+\mathrm{T}$ cell responses being twice as frequent as $\mathrm{CD} 8 \mathrm{~T}$ cell responses at 6 months. In addition, the predominant response was IL-2 within the CD4+ T cell subset. They also reported a decline in Th1 effector cells over time. Finally, the magnitude of virus-specific T cell responses correlated with peak antibody responses at 6 months.

These results are similar to a recently published similar study of 206 COVID-19+ patients (Sekine et. al., Cell 2020) with mild to severe disease. Their study demonstrated that SARS-CoV-2-specific CD4+ T cells primarily expressed IFN-g, IL-2, and TNF while SARS-CoV-2-specific CD8+ T cells mostly expressed IFN-g; the study also demonstrated that antibody responses tracked closely with virusspecific $\mathrm{T}$ cell responses. The current study is smaller, uses slightly less rigorous methods (virusspecific responses are assessed without tetramer-staining assays), and demonstrates similar findings of correlated $\mathrm{T}$ cell and antibody responses and adds to the literature, although not necessarily novel data.

Comments:

1. The data does not report correlation with magnitude and duration of SARS-CoV-2 viremia (recent data suggests that patients with severe COVID have prolonged nasopharyngeal viral loads). If viral load data is available, $\mathrm{Ct}$ values might be used to correlate with viremia (although acknowledging that these values are not clearly linked to infectivity per se).

2. A very large number of the study participants were not asymptomatic; this calls into question the generalizability of the results, and the paper reports little clinical follow up data. Whether the initial 
robust $\mathrm{T}$ cell responses protected against more severe forms of COVID-19 is unclear.

3. The authors do not appear to have used tetramer-staining to determine SARS-CoV-2 specific responses; this should be included in the Discussion.

4. The authors reported that virus $M / N$ protein-specific $T$ cell responses were equally high as virus spike protein-specific responses. Do the authors think that this might be due to the milder clinical phenotype of their study population?

5. As this paper is not the first to report long-term T cell responses, the second sentence of the Discussion should be revised and comparisons to previously reported paper(s) should be given. 